\title{
Physical and nutritional qualities of eggs and meats fed shrimp head meal to layer chicken
}

\author{
S Yeasmin ${ }^{1}$, MS Islam ${ }^{2}$, SD Nath ${ }^{1}$ and SS Islam ${ }^{2 *}$
}

${ }^{1}$ Student, Agrotechnology Discipline, Khulna University, Khulna-9208, Bangladesh; ${ }^{2}$ Professor, Agrotechnology Discipline, Khulna University, Khulna-9208, Bangladesh

\begin{abstract}
The experiment was conducted to investigate the effects of substitution of soybean meal of laying hens ration by shrimp head meal (SHM) on physical and nutritional quality of eggs and meats. Three hundred Hisex White laying hens were divided into five treatment groups and allocated five experimental diets included different levels of SHM. Soybean meal contents of control ration was substituted by SHM meal at the rate of 25, 50, 75 and $100 \%$, respectively. Samples of SHM, eggs and meats were subjected to proximate analysis. Physical properties of eggs and meats were also analyzed following the standard procedures. Highest CP contents (\%) of eggs were recorded to be $11.39 \pm 0.27$ and $10.83 \pm 0.18$ at initial and peak production periods, respectively in laying hens group fed ration substituted SBM by SHM at the rate of $25 \%$. Significantly $(p<0.001)$ highest value of redness $\left(a^{*}\right)$ of egg yolk was recorded to be 1.39 in laying hens group fed diet where SBM was completely substituted by SHM and lowest to be -3.11 in control group (no substitution) at initial production stage $\left(18^{\text {th }}\right.$ to $20^{\text {th }}$ week). Significantly $(p=0.05)$ highest CP $(\%)$ contents of meats was found to be $19.37 \pm 0.36$ in laying hens fed diets substituted SBM at the rate of $75 \%$ by SHM and lowest in complete substituted group. It can be concluded that substitution of soybean meal of laying hens ration at the rate of $25 \%$ by SHM is suitable for better egg and meat quality.
\end{abstract}

Keywords: alternate protein source, carcass parts, egg weight, meat color, yolk color

\section{Introduction}

Poultry is one of the fastest growing and most promising industries with the brightest future for Bangladesh. Following a high population growth, urbanization and demand elasticity, the demand for poultry products is expected to increase in the future. Feed cost which accounts for $65-75 \%$ of the total cost of poultry egg and meat production remains the major factor limiting the development and expansion of poultry farming (Kirkpinar and Acikgoz, 2018). Due to the unavailability and high cost of poultry feed particularly protein sources inhabit the formulation of least cost ration. The bulk of the feed cost arises from protein concentrates such as fishmeal, soybean meal and groundnut cake. Prices of these conventional protein sources have risen so high in recent times that it is not economical to use them in poultry feeds which have necessitated the search for alternatives to the expensive protein concentrates (Adeyemi, 2005). Animal nutritionists have therefore come to the conclusion that replacement of expensive conventional feed ingredients with cheap and available substitutes represents a suitable strategy at reducing feed cost and encouraging production. Many research efforts were taken in the search for alternatives to soybean meal in poultry diets. As a result poultry nutritionists have been working with various types of unconventional feed sources (Ani and Okorie, 2009).

It was estimated that 248.8 metric tons of shrimp waste is produced daily in the shrimp processing industries located in the coastal region of Bangladesh which represents $37 \%$ of total shrimp mass received by the industry (Hossain et al., 2018). This waste product from the shrimp processing plants has the potential of being an alternative protein source in layer rations, partially or totally replacing conventional protein sources such as soybean meal (SBM), meat and bone meal and fish meal. Head meal of black tiger shrimp (Penaeus monodon) contains an average of $52.3 \%$ crude protein, $6.4 \%$ ether extract, $10.8 \%$ crude fiber and $20.4 \%$ crude ash (Rahman and Koh, 2014). Gernat (2001) conducted an experiment and found that SHM had no significant effect on egg weight and specific gravity. Researchers suggest that shrimp meal contains high levels of $\mathrm{Ca}$ (Rahman and 
Koh, 2014) and carotenoid pigment (astaxanthin) (Gernat, 2001) which can improve the egg shell quality and yolk color, respectively. The eggshell strength and yolk color of chicken eggs was significantly $(p<0.05)$ increased with the increasing levels of dietary shrimp meal (Rahman, 2016). According to Rahman (2016) the dressing yield was not varied significantly $(p>0.05)$ due the inclusion of shrimp meal in the diet which was compatible with the findings of Fanimo et al. (1996). Similarly, shrimp meal had no significant effect on percentage of giblets yield among the dietary treatment groups $(p>0.05)$ (Rahman, 2016). Therefore, the research was undertaken to investigate the effect of different levels of shrimp head meal on physical and nutritional quality of eggs and meats of laying hens.

\section{Materials and Methods}

\section{Experimental site, design and laying hens}

The experiment was conducted at Dr. Purnendu Gain Field Laboratory, Agrotechnology Discipline, Khulna University, Khulna, Bangladesh. The design of the experiment was based on completely randomized design (CRD). Hisex White laying hens were divided into five treatment groups and assigned at random to five different diets included different levels of shrimp head meal (SHM). There were 3 replications for each treatment and the number of birds under each replication was 20 . Therefore, 60 birds were kept under each treatment and total number of birds was 300 .

\section{Management practices}

The experimental birds were kept in a shed having slate floor. The floor as well as feeders and waterers were cleaned regularly. Proper biosecurity measures were taken during the experimental period. The experimental birds were debeaked earlier at 70 days of bird's age using electrical debeaker. During the experimental period from $18^{\text {th }}$ week to $34^{\text {th }}$ weeks of laying hens age, 16 hours lighting period and 8 hours dark period was maintained properly. During laying period to prevent Newcastle disease a live vaccine (Avinew) was applied regularly every 2 months interval and killed vaccine against Newcastle disease (Imopest) was also applied 5 months interval. Fowl cholera vaccine was applied during laying period. Birds were also vaccinated earlier (before 18 weeks of age) against all infectious diseases such as Newcastle disease, infectious bursal disease, Marek's, fowl pox, salmonella, infectious laryngotracheitis, fowl cholera and egg drop syndrome according to the recommendation of the vaccine manufacturer. Deworming and medication against coccidiosis was provided routinely. All birds were kept in the similar environment and uniform management was allowed to all the birds.

Table 1: Proximate composition of shrimp head meal (\% on DM basis)

\begin{tabular}{lcc}
\hline \multicolumn{1}{c}{$\begin{array}{c}\text { Proximate } \\
\text { components (\%) }\end{array}$} & \multicolumn{2}{c}{ Shrimp species } \\
\cline { 2 - 3 } & $\begin{array}{c}\text { Black tiger } \\
\text { shrimp } \\
\text { (Penaeus } \\
\text { monodon) }\end{array}$ & $\begin{array}{c}\text { Giant } \\
\text { freshwater } \\
\text { prawn } \\
\text { (Macrobrach } \\
\text { ium } \\
\text { rosenbergii) }\end{array}$ \\
\hline Dry matter (DM) & 22.61 & 45.39 \\
Crude protein (CP) & 52.26 & 32.34 \\
Total ash (TA) & 21.69 & 17.51 \\
Acid insoluble ash (AIA) & 0.59 & 0.62 \\
Crude fibre (CF) & 3.20 & 4.10 \\
Ether extract (EE) & 5.78 & 24.23 \\
\hline
\end{tabular}

Preparation and proximate analysis of shrimp head meal (SHM), eggs and meats

Heads of black tiger shrimp (Penaeus monodon) were collected from shrimp processing plants. After arrival of shrimp heads in the experimental site it was allowed to sundry for three consecutive days. After drying the shrimp heads were crushed by a grinding machine. Proximate components (DM, CP, CF, EE and ash contents) of the shrimp head meal (SHM) was estimated in the Animal Husbandry Laboratory of Agrotechnology Discipline, Khulna University, Bangladesh following the method of AOAC (2005). Proximate composition of head from two major species of shrimp such as giant freshwater prawn (Macrobrachium rosenbergii) and black tiger (Penaeus monodon) was determined separately (Table 1 ). Proximate composition of eggs and meats of laying hens under different treatments were also estimated following the same procedures.

\section{Ration formulation and feeding system}

After weighing, required quantity of feed ingredients and feed additives were mixed homogeneously using a feed mixing machine. In the experimental rations, main protein source (SBM) was substituted by SHM at the rate of 0 , 
$25,50,75$ and $100 \%$, respectively. Other ingredients of five experimental rations were kept in constant proportions (Table 2). Feeds and water were supplied ad libitum to the experimental laying hens two times daily first in the morning at $7.30 \mathrm{am}$ and second in the evening at $4.00 \mathrm{pm}$. Laying hens under all treatment groups fed isocaloric diets.

\section{Physical traits and color determination}

Physical traits of eggs and meats were determined according to Singh (1990). Meat samples were standardized into two $2.54 \mathrm{~cm}$ thick steak samples (AMSA, 1995) for objective color evaluation ( $L^{*}, a^{*}, b^{*}, c^{*}$ and $h^{*}$ ). Before data collection the instrument was calibrated with a white calibration plate $\left(L^{*}=97.06, a^{*}=-0.14\right.$ $b^{*}=1.93$, ) covered in the same film wrapping the samples. Data were collected in CIE L*, a*, b* color space through the meat film. Lightness $\left(L^{*}\right)$, redness $\left(a^{*}\right)$, yellowness $\left(b^{*}\right)$ chroma [or color saturation, ða_2 $p$ b_2p 0:5], and hue angle [arctangent $\left.\left(b^{*} / a^{*}\right) 360 \_/\left(2 \_3.14\right)\right]$ were evaluated. Breast muscle and drumstick color coordinates ( $L^{*}, a^{*}$ and $\left.b^{*}\right)$ were recorded with a digital Minolta CR300 chromometer (Minolta Co., Osaka, Japan) on the surface exposed by cutting. Coordinate $a *$ ranged from red $(+a *)$ to green ($\left.a^{*}\right)$ and coordinate $b^{*}$ from yellow ( $\left.+b^{*}\right)$ to blue (-b*) (Hunterlab, 1996). Three readings of $L^{*}$, $a^{*}, b^{*}, c^{*}$ and $h^{*}$ values were obtained at different sites. Egg yolk color was also estimated in similar ways.

\section{Data collection and statistical analysis}

Egg weight data were recorded weekly and body weight at fortnightly from each pen. The data of egg weight were collected from the average weight of at least 10 eggs from each pen and the data of body weight of laying hens were collected from the average body weight of at least 5 birds. The data were analyzed using the GLM procedure of SAS version 9.1.3 (SAS, 2009). Effects of shrimp head meal were tested by analysis of variance and DMRT was used to compare the treatment means, with significance level considered at $p<0.05$.

Table 2: Composition $(\mathrm{Kg} / 100 \mathrm{Kg})$ of experimental diets under different treatments

\begin{tabular}{|c|c|c|c|c|c|}
\hline \multirow{2}{*}{ Ingredients $(\mathrm{Kg} / 100 \mathrm{Kg})$} & \multicolumn{5}{|c|}{ Rate of substitution of SBM by SHM (\%) } \\
\hline & $\mathbf{0}$ & 25 & 50 & 75 & 100 \\
\hline Maize (Zea mays) & 50.00 & 50.00 & 50.00 & 50.00 & 50.00 \\
\hline Rice polish (Oryza sativa) & 8.90 & 8.90 & 8.90 & 8.90 & 8.90 \\
\hline Wheat bran (Triticum aestivum) & 4.000 & 4.00 & 4.00 & 4.00 & 4.00 \\
\hline Soybean meal (SBM) & 22.00 & 16.50 & 11.00 & 5.50 & 00 \\
\hline Shrimp head meal (SHM) & 00 & 5.50 & 11.00 & 16.50 & 22.00 \\
\hline Protein concentrate & 5.00 & 5.00 & 5.00 & 5.00 & 5.00 \\
\hline Limestone & 9.00 & 9.00 & 9.00 & 9.00 & 9.00 \\
\hline Ascovit poultry VM (vitamin) & 0.125 & 0.125 & 0.125 & 0.125 & 0.125 \\
\hline Common salt & 0.200 & 0.200 & 0.200 & 0.200 & 0.200 \\
\hline DL-methionine & 0.125 & 0.125 & 0.125 & 0.125 & 0.125 \\
\hline ADM - lysine & 0.050 & 0.050 & 0.050 & 0.050 & 0.050 \\
\hline Sodium bi carbonate & 0.050 & 0.050 & 0.050 & 0.050 & 0.050 \\
\hline Choline chloride & 0.100 & 0.100 & 0.100 & 0.100 & 0.100 \\
\hline Klinofeed plus (Mycotoxin binder) & 0.200 & 0.200 & 0.200 & 0.200 & 0.200 \\
\hline Rovabio ${ }^{\circledR}$ Max (Enzyme) & 0.020 & 0.020 & 0.020 & 0.020 & 0.020 \\
\hline Bioacid (anti-salmonela) & 0.200 & 0.200 & 0.200 & 0.200 & 0.200 \\
\hline Hedox dry (Antioxidant) & 0.020 & 0.020 & 0.020 & 0.020 & 0.020 \\
\hline Probiolac (probiotics) & 0.010 & 0.010 & 0.010 & 0.010 & 0.010 \\
\hline Total & 100 & 100 & 100 & 100 & 100 \\
\hline Energy content $(\mathrm{Kcal} / \mathrm{kg})$ & 2734.00 & 2734.20 & 2734.40 & 2734.60 & 2734.80 \\
\hline Protein content $(\mathrm{g} / 100 \mathrm{~g})$ & 18.86 & 19.03 & 19.20 & 19.37 & 19.54 \\
\hline
\end{tabular}




\section{Results and Discussion}

\section{Physical properties of eggs}

Data of the Table 3 showed that different physical traits like egg weight, specific gravity, haugh unit, shape index, albumen weight, albumen index, yolk index, shell weight and shell thickness were not varied significantly $(p>0.05)$ due to the inclusion of shrimp head meal (SHM) in laying hens ration at the initial production stage $\left(18^{\text {th }}\right.$ to $20^{\text {th }}$ weeks). Yolk weight ( $\mathrm{g} / \mathrm{egg}$ ) was found highest $(p<0.01)$ in laying hens fed control diets $(11.87 \pm 0.36)$ and lowest in laying hens group fed diets substituted soybean meal (SBM) by SHM at the rate $25 \%(10.05 \pm 0.35)$ at initial production stage. Shrimp head meal had a highly significant effect $(p<0.001)$ on albumin index at peak production period ( $28^{\text {th }}$ to $34^{\text {th }}$ weeks) being highest $(11.87 \%)$ in laying hens group fed diets substituted soybean meal (SBM) by SHM at the rate of $75 \%$ and lowest $(7.68 \%)$ in $50 \%$ substitution group. Rest physical traits under study were statistically similar $(p>0.05)$ for all treatment groups at peak production period $\left(28^{\text {th }}\right.$ to $34^{\text {th }}$ weeks). Inclusion of shrimp meal in laying hens ration had no significant effects $(p<0.05)$ on egg weight, shell thickness and specific gravity (Rahman, 2016) which is consistent with the present findings. No significant effects of SHM in laying hens ration on egg weight and specific gravity was also reported by Gernat (2001). Another study suggests that shrimp meal contains high levels of Ca which can improve the egg shell (Rahman and Koh, 2014). However, in present study, eggshell weight and thickness were not differed significantly $(p>0.05)$ among diet groups included different levels of SHM.

Table 3: Physical traits of the eggs (Mean \pm SE) of laying hens under different dietary treatments

\begin{tabular}{|c|c|c|c|c|c|c|}
\hline \multirow[t]{2}{*}{ Parameters } & \multicolumn{5}{|c|}{ Rate of substitution of SBM by SHM (\%) } & \multirow{2}{*}{$\begin{array}{c}\mathbf{P} \\
\text { value }\end{array}$} \\
\hline & $\mathbf{0}$ & 25 & 50 & 75 & 100 & \\
\hline \multicolumn{7}{|c|}{ Initial production stage $\left(18^{\text {th }}\right.$ to $20^{\text {th }}$ weeks $)$} \\
\hline Egg weight (g/egg) & $47.76 \pm 1.68$ & $45.29 \pm 2.44$ & $45.97 \pm 0.99$ & $42.78 \pm 1.61$ & $44.13 \pm 0.51$ & 0.30 \\
\hline $\begin{array}{l}\text { Specific gravity of } \\
\text { eggs }(\mathrm{g} / \mathrm{ml})\end{array}$ & $1.14 \pm 0.02$ & $1.13 \pm 0.04$ & $1.13 \pm 0.01$ & $1.10 \pm 0.02$ & $1.10 \pm 0.00$ & 0.76 \\
\hline Haugh unit & $92.06 \pm 0.41$ & $92.43 \pm 1.06$ & $91.92 \pm 0.41$ & $92.58 \pm 0.71$ & $91.40 \pm 0.72$ & 0.78 \\
\hline Shape index (\%) & $65.31 \pm 2.23$ & $67.94 \pm 0.59$ & $65.96 \pm 1.77$ & $64.52 \pm 2.59$ & $66.05 \pm 0.33$ & 0.72 \\
\hline $\begin{array}{l}\text { Yolk weight } \\
\text { (g/yolk) }\end{array}$ & $11.87^{\mathrm{a}} \pm 0.36$ & $10.05^{\mathrm{b}} \pm 0.35$ & $10.85^{\mathrm{ab}} \pm 0.13$ & $11.09^{\mathrm{ab}} \pm 0.37$ & $10.30^{b} \pm 0.32$ & 0.01 \\
\hline $\begin{array}{l}\text { Albumin weight } \\
\text { (g/egg) }\end{array}$ & $29.18 \pm 1.28$ & $28.05 \pm 1.04$ & $28.10 \pm 0.94$ & $25.98 \pm 0.54$ & $27.25 \pm 1.11$ & 0.31 \\
\hline Albumin index (\%) & $10.76 \pm 0.38$ & $11.50 \pm 0.68$ & $10.77 \pm 0.98$ & $11.20 \pm 0.51$ & $9.65 \pm 0.35$ & 0.35 \\
\hline Yolk index (\%) & $40.96 \pm 0.44$ & $43.54 \pm 1.29$ & $42.18 \pm 1.55$ & $44.37 \pm 0.90$ & $42.50 \pm 0.27$ & 0.23 \\
\hline $\begin{array}{l}\text { Eggshell weight } \\
\text { (g/egg) }\end{array}$ & $4.83 \pm 0.14$ & $4.83 \pm 0.34$ & $4.89 \pm 0.18$ & $4.65 \pm 0.18$ & $4.80 \pm 0.10$ & 0.93 \\
\hline $\begin{array}{l}\text { Eggshell thickness } \\
(\mathrm{mm})\end{array}$ & $0.38 \pm 0.00$ & $0.39 \pm 0.01$ & $0.39 \pm 0.00$ & $0.38 \pm 0.00$ & $0.40 \pm 0.00$ & 0.42 \\
\hline \multicolumn{7}{|c|}{ Peak production stage $\left(28^{\text {th }}\right.$ to $34^{\text {th }}$ weeks) } \\
\hline Egg weight (g/egg) & $56.13 \pm 1.32$ & $55.34 \pm 2.51$ & $54.22 \pm 1.70$ & $53.52 \pm 0.91$ & $52.24 \pm 2.71$ & 0.66 \\
\hline $\begin{array}{l}\text { Specific gravity of } \\
\text { eggs }(\mathrm{g} / \mathrm{ml})\end{array}$ & $1.14 \pm 0.00$ & $1.08 \pm 0.01$ & $1.15 \pm 0.01$ & $1.14 \pm 0.02$ & $1.12 \pm 0.02$ & 0.10 \\
\hline Haugh unit & $90.56 \pm 0.39$ & $90.61 \pm 0.58$ & $91.70 \pm 0.55$ & $91.58 \pm 0.10$ & $91.44 \pm 0.68$ & 0.37 \\
\hline Shape index (\%) & $77.77 \pm 0.77$ & $74.86 \pm 2.63$ & $75.86 \pm 0.37$ & $77.50 \pm 0.65$ & $77.35 \pm 0.16$ & 0.46 \\
\hline $\begin{array}{l}\text { Yolk weight } \\
\text { (g/yolk) }\end{array}$ & $15.83 \pm 0.25$ & $15.29 \pm 1.14$ & $15.81 \pm 0.90$ & $15.05 \pm 0.48$ & $15.12 \pm 1.25$ & 0.94 \\
\hline $\begin{array}{l}\text { Albumin weight } \\
\text { (g/egg) }\end{array}$ & $31.98 \pm 0.92$ & $31.71 \pm 1.34$ & $30.34 \pm 1.82$ & $31.46 \pm 1.66$ & $29.45 \pm 1.36$ & 0.71 \\
\hline Albumin index (\%) & $8.37^{b} \pm 0.74$ & $7.68^{b} \pm 0.21$ & $11.18^{\mathrm{a}} \pm 0.23$ & $11.87^{\mathrm{a}} \pm 0.42$ & $10.91^{a} \pm 0.93$ & 0.001 \\
\hline Yolk index (\%) & $43.33 \pm 2.23$ & $41.70 \pm 0.34$ & $46.27 \pm 0.66$ & $44.48 \pm 0.86$ & $45.86 \pm 1.56$ & 0.06 \\
\hline $\begin{array}{l}\text { Eggshell weight } \\
\text { (g/egg) }\end{array}$ & $5.16 \pm 0.06$ & $5.35 \pm 0.27$ & $5.27 \pm 0.09$ & $5.37 \pm 0.05$ & $5.13 \pm 0.07$ & 0.68 \\
\hline $\begin{array}{l}\text { Eggshell thickness } \\
(\mathrm{mm})\end{array}$ & $0.40 \pm 0.00$ & $0.41 \pm 0.01$ & $0.39 \pm 0.00$ & $0.42 \pm 0.00$ & $0.39 \pm 0.01$ & 0.37 \\
\hline
\end{tabular}




\section{Shrimp head meal in layer chicken ration}

Table 4: Proximate composition of edible portion of eggs (Mean \pm SE) of laying hens under different dietary treatments

\begin{tabular}{|c|c|c|c|c|c|c|}
\hline \multirow{2}{*}{$\begin{array}{l}\text { Proximate } \\
\text { components } \\
\text { (DM basis) }\end{array}$} & \multicolumn{5}{|c|}{ Rate of substitution of SBM by SHM (\%) } & \multirow[t]{2}{*}{ P value } \\
\hline & $\mathbf{0}$ & 25 & 50 & 75 & 100 & \\
\hline \multicolumn{7}{|c|}{ Initial production stage $\left(18^{\text {th }}\right.$ to $20^{\text {th }}$ weeks) } \\
\hline Dry matter (\%) & $21.76 \pm 0.42$ & $22.25 \pm 0.78$ & $22.41 \pm 0.75$ & $22.12 \pm 0.28$ & $21.58 \pm 0.96$ & 0.90 \\
\hline Crude protein (\%) & $10.69^{\mathrm{ab}} \pm 0.08$ & $11.39^{\mathrm{a}} \pm 0.27$ & $10.77^{\mathrm{ab}} \pm 0.10$ & $9.29^{c} \pm 0.08$ & $10.26^{\mathrm{b}} \pm 0.39$ & 0.0008 \\
\hline Ether extract (\%) & $8.35 \pm 0.05$ & $8.45 \pm 0.56$ & $8.64 \pm 0.98$ & $9.64 \pm 0.28$ & $8.67 \pm 0.46$ & 0.53 \\
\hline Total ash (\%) & $1.08 \pm 0.00$ & $1.15 \pm 0.01$ & $1.11 \pm 0.04$ & $1.06 \pm 0.02$ & $1.12 \pm 0.01$ & 0.17 \\
\hline \multicolumn{7}{|c|}{ Peak production stage $\left(28^{\text {th }}\right.$ to $34^{\text {th }}$ weeks) } \\
\hline Dry matter $(\%)$ & $21.90 \pm 0.26$ & $21.75 \pm 0.51$ & $21.83 \pm 0.37$ & $20.43 \pm 0.58$ & $20.60 \pm 0.36$ & 0.08 \\
\hline Crude protein (\%) & $10.52^{\mathrm{a}} \pm 0.03$ & $10.83^{\mathrm{a}} \pm 0.18$ & $10.63^{\mathrm{a}} \pm 0.10$ & $9.62^{b} \pm 0.24$ & $8.68^{\mathrm{c}} \pm 0.08$ & $<0.0001$ \\
\hline Ether extract (\%) & $8.46^{b} \pm 0.17$ & $8.26^{b} \pm 0.12$ & $9.10^{\mathrm{a}} \pm 0.23$ & $8.26^{b} \pm 0.12$ & $9.00^{\mathrm{a}} \pm 0.15$ & 0.01 \\
\hline Total ash (\%) & $1.09 \pm 0.03$ & $1.10 \pm 0.02$ & $1.04 \pm 0.01$ & $1.15 \pm 0.02$ & $1.10 \pm 0.02$ & 0.11 \\
\hline
\end{tabular}

$a, b, c$ Values in the same row bearing different superscripts are significantly different. SBM, Soybean meal; SHM, Shrimp head meal; $P$ values indicate significance level.

\section{Proximate composition of eggs}

Crude protein contents of eggs varied significantly among the laying hens groups fed diets included different levels of SHM at both initial and peak production stages (Table 4). Highest CP contents (\%) were recorded to be $11.39 \pm 0.27$ and $10.83 \pm 0.18$ at initial $\left(18^{\text {th }}\right.$ to $20^{\text {th }}$ weeks) and peak ( $28^{\text {th }}$ to $34^{\text {th }}$ weeks) production stages, respectively in laying hens group fed ration substituted SBM by SHM at the rate of $25 \%$. Dry matter and total ash contents showed no significant variation $(p>0.05)$ for both production stages. However, ether extract contents varied significantly $(p=0.01)$ among different treatment groups at peak production stage $\left(28^{\text {th }}\right.$ to $34^{\text {th }}$ weeks) where the highest $\mathrm{EE}$ content of eggs was recorded to be $9.10 \pm 0.23$ in laying hens group fed diets having $50 \%$ substitution.

Table 5: Color measurement of egg yolk (Mean \pm SE) of laying hens under different dietary treatments

\begin{tabular}{|c|c|c|c|c|c|c|}
\hline \multirow{2}{*}{$\begin{array}{c}\text { Color } \\
\text { indicators }\end{array}$} & \multicolumn{5}{|c|}{ Rate of substitution of SBM by SHM (\%) } & \multirow[t]{2}{*}{ P value } \\
\hline & $\mathbf{0}$ & 25 & 50 & 75 & 100 & \\
\hline \multicolumn{7}{|c|}{ Initial production stage $\left(18^{\text {th }}\right.$ to $20^{\text {th }}$ weeks) } \\
\hline Lightness $\left(\mathrm{L}^{*}\right)$ & $51.01 \pm 2.01$ & $47.30 \pm 0.71$ & $47.95 \pm 0.57$ & $52.52 \pm 1.70$ & $48.30 \pm 2.58$ & 0.21 \\
\hline Redness $\left(a^{*}\right)$ & $-3.11^{\mathrm{c}} \pm 0.11$ & $0.50^{\mathrm{ab}} \pm 0.14$ & $-0.65^{b} \pm 0.59$ & $0.17^{\mathrm{ab}} \pm 0.74$ & $1.39^{\mathrm{a}} \pm 0.13$ & 0.0003 \\
\hline Yellowness $\left(b^{*}\right)$ & $36.49 \pm 1.34$ & $34.92 \pm 0.95$ & $33.67 \pm 0.71$ & $39.70 \pm 2.82$ & $36.43 \pm 2.21$ & 0.25 \\
\hline Chroma $\left(c^{*}\right)$ & $36.71 \pm 0.85$ & $35.26 \pm 1.28$ & $33.69 \pm 0.71$ & $39.72 \pm 2.81$ & $36.46 \pm 2.22$ & 0.25 \\
\hline Hue angle $\left(h^{*}\right)$ & $95.20^{\mathrm{a}} \pm 0.33$ & $90.82^{\mathrm{b}} \pm 0.22$ & $91.08^{\mathrm{b}} \pm 1.00$ & $89.90^{\mathrm{bc}} \pm 1.14$ & $87.81^{c} \pm 0.18$ & 0.0004 \\
\hline \multicolumn{7}{|c|}{ Peak production stage $\left(28^{\text {th }}\right.$ to $34^{\text {th }}$ weeks) } \\
\hline Lightness $\left(L^{*}\right)$ & $55.50 \pm 1.76$ & $51.25 \pm 0.75$ & $50.82 \pm 0.48$ & $55.92 \pm 1.34$ & $51.3 \pm 2.31$ & 0.08 \\
\hline Redness $\left(a^{*}\right)$ & $-5.69 \pm 0.63$ & $0.55 \pm 0.13$ & $-0.67 \pm 0.63$ & $0.17 \pm 0.78$ & $1.44 \pm 0.12$ & 0.08 \\
\hline Yellowness $\left(b^{*}\right)$ & $39.34 \pm 1.51$ & $39.08 \pm 1.27$ & $36.34 \pm 0.98$ & $42.56 \pm 2.53$ & $39.35 \pm 2.25$ & 0.27 \\
\hline Chroma $\left(c^{*}\right)$ & $35.85 \pm 1.03$ & $39.01 \pm 1.50$ & $36.44 \pm 0.49$ & $42.65 \pm 2.46$ & $39.85 \pm 2.34$ & 0.10 \\
\hline Hue angle $\left(\mathrm{h}^{*}\right)$ & $69.28 \pm 29.41$ & $94.12 \pm 0.07$ & $94.70 \pm 0.79$ & $92.60 \pm 1.29$ & $91.24 \pm 0.37$ & 0.62 \\
\hline
\end{tabular}

$a, b, c$ Values in the same row bearing different superscripts are significantly different. SBM, Soybean meal; SHM, Shrimp head meal; P values indicate significance level. 


\section{Color of egg yolk}

Color measurements of egg yolk of laying hens fed diets included different levels of SHM is shown in Table 5. At the initial production stage ( $18^{\text {th }}$ to $20^{\text {th }}$ weeks), shrimp head meal (SHM) had a significant effect $(p<0.001)$ on redness $\left(a^{*}\right)$ and hue angle $\left(h^{*}\right)$ of egg yolk. Significantly $(p<0.001)$ highest value of redness $\left(a^{*}\right)$ was recorded to be 1.39 in laying hens group fed diet where SBM was completely substituted by SHM and lowest to be -3.11 in control group (no substitution) at initial production stage. Other color indicators of egg yolk like lightness $\left(L^{*}\right)$, yellowness $\left(b^{*}\right)$ and chroma $\left(c^{*}\right)$ were not varied significantly among different treatment groups in initial production period. On the other hand, all color measurement indicators studied for egg yolk did not show any significant variation among treatment groups at peak production stage $\left(28^{\text {th }}\right.$ to $34^{\text {th }}$ weeks). Highest value of redness $\left(a^{*}\right)$ of egg yolk in complete substituted group (1.39 $\pm 0.13)$ and lowest in control group $(-3.11 \pm 0.11)$ in the present study is due to the carotenoid pigment (astaxanthin) contents of SHM, because it is well known that this pigment can increase the yolk color (Anderson et al., 2008). In a study, yolk color of chicken eggs was significantly $(p<0.05)$ increased with the increasing levels of dietary shrimp meal in the diets (Rahman, 2016)

Table 6: Weight measurement of different carcass parts of layer meats (Mean \pm SE) under different dietary treatments

\begin{tabular}{|c|c|c|c|c|c|c|}
\hline \multirow[t]{2}{*}{ Parameters } & \multicolumn{5}{|c|}{ Rate of substitution of SBM by SHM (\%) } & \multirow[t]{2}{*}{ P value } \\
\hline & $\mathbf{0}$ & 25 & & $\mathbf{0}$ & 100 & \\
\hline \multicolumn{7}{|c|}{ Initial production stage $\left(18^{\text {th }}\right.$ to $20^{\text {th }}$ weeks) } \\
\hline Live weight (kg/bird) & $1.50^{\mathrm{a}} \pm 0.01$ & $1.17^{\mathrm{d}} \pm 0.01$ & $1.23^{c} \pm 0.00$ & $1.37^{\mathrm{b}} \pm 0.02$ & $1.13^{\mathrm{d}} \pm 0.01$ & $<.0001$ \\
\hline \multirow{2}{*}{$\begin{array}{l}\text { Dressed weight } \\
\text { (g/bird) }\end{array}$} & $1144.00^{\mathrm{a}}$ & $906.67^{\mathrm{bc}}$ & $960.16^{\mathrm{b}}$ & $1093.17^{a}$ & $853.66^{c}$ & .0002 \\
\hline & \pm 6.65 & \pm 21.27 & \pm 15.33 & \pm 58.41 & \pm 20.64 & \\
\hline Neck weight (g/bird) & $35.20^{\mathrm{a}} \pm 0.61$ & $33.73^{a} \pm 0.69$ & $34.26^{\mathrm{a}} \pm 0.33$ & $26.73^{\mathrm{c}} \pm 0.49$ & $30.83^{b} \pm 0.20$ & $<.0001$ \\
\hline $\begin{array}{l}\text { Full wing weight } \\
\text { (g/bird) }\end{array}$ & $49.06^{\mathrm{a}} \pm 0.35$ & $48.03^{b} \pm 0.08$ & $45.83^{c d} \pm 0.27$ & $45.53^{d} \pm 0.37$ & $46.70^{c} \pm 0.30$ & $<.0001$ \\
\hline Thing weight ( $\mathrm{g} / \mathrm{bird}$ ) & $69.30^{\mathrm{a}} \pm 0.41$ & $48.26^{d} \pm 0.18$ & $53.23^{b} \pm 0.38$ & $51.23^{\mathrm{C}} \pm 0.48$ & $48.00^{d} \pm 1.05$ & $<.0001$ \\
\hline \multirow[t]{2}{*}{ Back weight (g/bird) } & $182.66^{\mathrm{a}}$ & $166.83^{b}$ & $174.33^{\mathrm{ab}}$ & $139.00^{c}$ & $170.40^{\mathrm{ab}}$ & .0006 \\
\hline & \pm 1.85 & \pm 6.19 & \pm 0.33 & \pm 2.08 & \pm 7.70 & \\
\hline \multirow{2}{*}{$\begin{array}{l}\text { Breast weight } \\
\text { (g/bird) }\end{array}$} & $248.33^{a}$ & $181.33^{d}$ & $208.00^{c}$ & $222.66^{\mathrm{b}}$ & $202.50^{c}$ & $<.0001$ \\
\hline & \pm 1.66 & \pm 2.33 & \pm 1.15 & \pm 5.04 & \pm 3.81 & \\
\hline $\begin{array}{l}\text { Drumstick weight } \\
\text { (g/bird) }\end{array}$ & $62.33^{\mathrm{a}} \pm 1.45$ & $48.66^{\mathrm{c}} \pm 0.44$ & $62.33^{\mathrm{a}} \pm 1.45$ & $55.00^{\mathrm{b}} \pm 2.88$ & $48.60^{\mathrm{C}} \pm 1.55$ & .0003 \\
\hline \multicolumn{7}{|c|}{ Peak production stage ( $28^{\text {th }}$ to $34^{\text {th }}$ weeks) } \\
\hline Live weight ( $\mathrm{kg} / \mathrm{bird})$ & $1.79^{a} \pm 0.02$ & $1.36^{\mathrm{c}} \pm 0.02$ & $1.56^{\mathrm{b}} \pm 0.02$ & $1.44^{\mathrm{c}} \pm 0.02$ & $1.54^{\mathrm{b}} \pm 0.02$ & $<.0001$ \\
\hline $\begin{array}{l}\text { Dressed weight } \\
(\mathrm{kg} / \text { bird })\end{array}$ & $1.44^{a} \pm 0.10$ & $1.07^{d} \pm 0.01$ & $1.27^{\mathrm{b}} \pm 0.01$ & $1.12^{\mathrm{C}} \pm 0.01$ & $1.24^{\mathrm{b}} \pm 0.01$ & $<.0001$ \\
\hline Neck weight (g/bird) & $34.00^{b} \pm 0.28$ & $32.56^{c} \pm 0.53$ & $32.53^{c} \pm 0.20$ & $33.5^{b c} \pm 0.17$ & $35.93^{\mathrm{a}} \pm 0.06$ & $<.0001$ \\
\hline $\begin{array}{l}\text { Full wing weight } \\
\text { (g/bird) }\end{array}$ & $52.51^{a} \pm 0.01$ & $46.38^{b} \pm 0.44$ & $45.50^{\complement} \pm 0.17$ & $46.73^{b} \pm 0.12$ & $52.23^{a} \pm 0.14$ & $<.0001$ \\
\hline $\begin{array}{l}\text { Wing tip weight } \\
\text { (g/bird) }\end{array}$ & $8.46^{a} \pm 0.03$ & $7.66^{\mathrm{b}} \pm 0.08$ & $7.69^{\mathrm{b}} \pm 0.02$ & $7.30^{c} \pm 0.15$ & $7.80^{\mathrm{b}} \pm 0.05$ & $<.0001$ \\
\hline Thing weight ( $\mathrm{g} / \mathrm{bird}$ ) & $69.70^{\mathrm{a}} \pm 0.25$ & $55.61^{\mathrm{e}} \pm 0.06$ & $60.23^{c} \pm 0.44$ & $59.00^{d} \pm 0.28$ & $65.53^{b} \pm 0.14$ & $<.0001$ \\
\hline \multirow[t]{2}{*}{ Back weight (g/bird) } & $214.73^{b}$ & $169.9^{d}$ & $219.01^{\mathrm{a}}$ & $197.83^{c}$ & $165.46^{\mathrm{e}} \pm 0.14$ & $<.0001$ \\
\hline & \pm 0.23 & \pm 0.20 & \pm 0.10 & \pm 0.32 & & \\
\hline \multirow{2}{*}{$\begin{array}{l}\text { Breast weight } \\
\text { (g/bird) }\end{array}$} & $264.66^{a}$ & $228.33^{b}$ & $206.08^{d}$ & $223.98^{c}$ & $206.90^{d} \pm 0.20$ & $<.0001$ \\
\hline & \pm 0.66 & \pm 0.12 & \pm 0.22 & \pm 0.18 & & \\
\hline $\begin{array}{l}\text { Drumstick weight } \\
\text { (g/bird) }\end{array}$ & $63.7^{a} \pm 0.20$ & $49.60^{d} \pm 0.26$ & $56.48^{\mathrm{C}} \pm 0.01$ & $48.98^{d} \pm 0.13$ & $59.33^{b} \pm 0.33$ & $<.0001$ \\
\hline
\end{tabular}




\section{Weight of carcass parts of layer meats}

Results revealed that the live weight, dressed weight and weights of other body parts were varied significantly due to the substitution of SBM of laying hens rations by different levels of SHM at both production stages (Table 6 ). The highest live weight of laying hens at initial egg production stage $\left(18^{\text {th }}\right.$ to $20^{\text {th }}$ weeks) was recorded to be $1.50 \mathrm{~kg} / \mathrm{bird}$ in control group and lowest to be $1.13 \mathrm{~kg} / \mathrm{bird}$ in complete substituted group. Dressed carcass weight also showed similar trends at initial production stage. Highest breast weight ( $\mathrm{g} / \mathrm{bird}$ ) was recorded to be $264.66 \pm 0.66$ in control group (no substitution) and lowest to be $206.08 \pm 0.22$ in diet group substituted SBM at the rate of $50 \%$ by SHM. Highest drumstick weight ( $g / b i r d)$ was also reported to be $63.7 \pm 0.20$ in control group. Rahman (2016) observed no significant effects of shrimp meal ( $p>0.05)$ on carcass traits among the dietary treatment groups. However, carcass traits varied significantly in the present study due the inclusion of SHM in laying hen's ration. Aktar et al. (2011) stated that dressed yield, thigh meat weight, breast meat weight and drumstick meat weight differed significantly due to substitute of fish meal of broiler ration by shrimp waste and marine waste. Similarly, significant variation observed in those traits due to inclusion of SHM in the diets in present study. According to Rahman (2016) the dressing yield was not varied significantly $(p>0.05)$ due the inclusion of shrimp meal in the diet which was compatible with the findings of Fanimo et al. (1996). In contrast, dressing yield was varied significantly in the present study.

\section{Weight of non-carcass parts of layer meat}

Weights of different non-carcass parts like feather, head, gizzard, heart, bile sac, lung and liver were varied significantly among different diets groups included different levels of SHM at both production stages (Table 7 ). The highest liver weight ( $\mathrm{g} /$ liver) was recorded to be $30.81 \pm 0.19$ in $75 \%$ SHM group and $43.29 \pm 0.00$ in complete substitution group at initial and peak production stages, respectively. The highest gizzard weights ( $\mathrm{g} /$ gizzard) were recorded to be $24.09 \pm 0.23$ and $24.87 \pm 0.01$ in laying hens groups feed $75 \%$ substituted and control diets at initial and peak stages of production, respectively.

Table 7: Weight measurement of different non-carcass parts of layer meats (Mean $\pm \mathrm{SE}$ ) under different dietary treatments

\begin{tabular}{|c|c|c|c|c|c|c|}
\hline \multirow[t]{2}{*}{ Parameters } & \multicolumn{5}{|c|}{ Rate of substitution of SBM by SHM (\%) } & \multirow[t]{2}{*}{ P value } \\
\hline & $\mathbf{0}$ & 25 & 50 & 75 & 100 & \\
\hline \multicolumn{7}{|c|}{ Initial production stage $\left(18^{\text {th }}\right.$ to $20^{\text {th }}$ weeks) } \\
\hline Feather weight $(\mathrm{g})$ & $\begin{array}{r}207.22^{\mathrm{b}} \\
\pm 3.43\end{array}$ & $\begin{array}{r}181.43^{\mathrm{c}} \\
\pm 5.52\end{array}$ & $\begin{array}{r}237.26^{\mathrm{a}} \\
\pm 1.50\end{array}$ & $\begin{array}{r}229.70^{\mathrm{a}} \\
\pm 2.97\end{array}$ & $\begin{array}{r}183.80^{c} \\
\pm 3.83\end{array}$ & $<.0001$ \\
\hline Head weight $(\mathrm{g})$ & $50.96^{\mathrm{a}} \pm 0.29$ & $38.80^{c} \pm 0.36$ & $39.76^{\mathrm{bc}} \pm 0.87$ & $40.76^{\mathrm{bc}} \pm 0.78$ & $41.46^{\mathrm{b}} \pm 0.20$ & $<.0001$ \\
\hline Gizzard weight (g) & $23.05^{\mathrm{ab}} \pm 0.81$ & $21.66^{\mathrm{b}} \pm 0.42$ & $23.92^{\mathrm{a}} \pm 0.14$ & $24.09^{\mathrm{a}} \pm 0.23$ & $21.68^{\mathrm{b}} \pm 0.08$ & 0.001 \\
\hline Heart weight $(\mathrm{g})$ & $6.50^{c} \pm 0.05$ & $6.10^{\mathrm{d}} \pm 0.14$ & $5.67^{e} \pm 0.04$ & $7.09^{b} \pm 0.09$ & $7.41^{\mathrm{a}} \pm 0.08$ & $<.0001$ \\
\hline $\begin{array}{l}\text { Bile with filled sac } \\
\text { weight }(g)\end{array}$ & $1.13^{\mathrm{bc}} \pm 0.01$ & $1.28^{\mathrm{bc}} \pm 0.01$ & $1.03^{\mathrm{c}} \pm 0.01$ & $1.93^{\mathrm{a}} \pm 0.17$ & $1.49^{b} \pm 0.20$ & 0.002 \\
\hline Lung weight $(\mathrm{g})$ & $7.53^{\mathrm{a}} \pm 0.01$ & $6.65^{c} \pm 0.05$ & $6.98^{\mathrm{b}} \pm 0.06$ & $5.84^{d} \pm 0.03$ & $7.66^{\mathrm{a}} \pm 0.10$ & $<.0001$ \\
\hline Liver weight $(\mathrm{g})$ & $29.83^{\mathrm{b}} \pm 0.19$ & $26.06^{\mathrm{c}} \pm 0.07$ & $20.46^{\mathrm{e}} \pm 0.32$ & $30.81^{\mathrm{a}} \pm 0.19$ & $21.71^{\mathrm{d}} \pm 0.39$ & $<.0001$ \\
\hline Shank weight $(\mathrm{g})$ & $25.00 \pm 0.28$ & $22.16 \pm 1.16$ & $23.26 \pm 0.49$ & $26.33 \pm 2.72$ & $21.76 \pm 0.93$ & 0.19 \\
\hline \multicolumn{7}{|c|}{ Peak production stage ( $28^{\text {th }}$ to $34^{\text {th }}$ weeks) } \\
\hline Feather weight $(\mathrm{g})$ & $\begin{array}{l}252.73 \\
a_{ \pm} \pm 0.39\end{array}$ & $\begin{array}{r}184.86 \\
a b \pm 0.18\end{array}$ & $\begin{array}{r}224.40^{\mathrm{a}} \\
\pm 0.40\end{array}$ & $\begin{array}{r}216.95^{\mathrm{a}} \\
\pm 0.62\end{array}$ & $\begin{array}{l}115.74^{b} \\
\pm 57.00\end{array}$ & 0.02 \\
\hline Head weight $(\mathrm{g})$ & $67.90^{\mathrm{a}} \pm 0.20$ & $56.50^{b} \pm 0.76$ & $50.56^{c} \pm 0.28$ & $55.46 \pm 0.26$ & $68.50^{\mathrm{a}} \pm 0.28$ & $<.0001$ \\
\hline Gizzard weight (g) & $24.87^{\mathrm{a}} \pm 0.01$ & $23.76^{\mathrm{b}} \pm 0.13$ & $22.25^{d} \pm 0.02$ & $23.39^{c} \pm 0.05$ & $24.83^{\mathrm{a}} \pm 0.04$ & $<.0001$ \\
\hline Heart weight $(\mathrm{g})$ & $7.87^{b} \pm 0.01$ & $6.36^{\mathrm{d}} \pm 0.00$ & $6.65^{c} \pm 0.02$ & $5.84^{e} \pm 0.02$ & $8.76^{a} \pm 0.02$ & $<.0001$ \\
\hline $\begin{array}{l}\text { Bile with filled sac } \\
\text { weight }(g)\end{array}$ & $2.76^{\mathrm{a}} \pm 0.00$ & $1.83^{\mathrm{d}} \pm 0.00$ & $2.66^{\mathrm{b}} \pm 0.00$ & $2.11^{\complement} \pm 0.01$ & $2.03^{c} \pm 0.06$ & $<.0001$ \\
\hline Lung weight (g) & $9.41^{\mathrm{a}} \pm 0.00$ & $7.53^{d} \pm 0.03$ & $8.74^{b} \pm 0.01$ & $7.14^{\mathrm{e}} \pm 0.01$ & $7.91^{c} \pm 0.01$ & $<.0001$ \\
\hline Liver weight $(\mathrm{g})$ & $42.28^{b} \pm 0.02$ & $39.69^{e} \pm 0.02$ & $40.72^{d} \pm 0.03$ & $41.20^{c} \pm 0.02$ & $43.29^{\mathrm{a}} \pm 0.00$ & $<.0001$ \\
\hline Shank weight (g) & $25.50^{\mathrm{a}} \pm 0.17$ & $22.33^{c} \pm 0.08$ & $23.13^{b} \pm 0.06$ & $23.51^{\mathrm{b}} \pm 0.15$ & $23.23^{b} \pm 0.14$ & $<.0001$ \\
\hline
\end{tabular}


Yeasmin et al. (2021) Bang. J. Anim. Sci. 50 (1):12-21

Table 8: Proximate composition of layer meats (Mean \pm SE) under different dietary treatments

\begin{tabular}{lcccccc}
\hline \multirow{2}{*}{$\begin{array}{c}\text { Proximate } \\
\text { components } \\
\text { (DM basis) }\end{array}$} & \multicolumn{4}{c}{ Rate of substitution of SBM by SHM (\%) } & P value \\
\cline { 2 - 6 } & $\mathbf{0}$ & $\mathbf{2 5}$ & $\mathbf{5 0}$ & $\mathbf{7 5}$ & $\mathbf{1 0 0}$ & \\
\hline Dry matter (\%) & $22.03 \pm 0.29$ & $20.63 \pm 0.58$ & $21.51 \pm 0.93$ & $22.50 \pm 0.35$ & $21.06 \pm 0.38$ & 0.21 \\
Crude protein (\%) & $18.33^{\mathrm{ab}} \pm 0.11$ & $17.79^{\mathrm{b}} \pm 0.33$ & $17.69^{\mathrm{b}} \pm 0.69$ & $19.37^{\mathrm{a}} \pm 0.36$ & $17.60^{\mathrm{b}} \pm 0.26$ & 0.05 \\
Ether extract (\%) & $1.26^{\mathrm{a}} \pm 0.03$ & $1.08^{\mathrm{b}} \pm 0.01$ & $1.25^{\mathrm{a}} \pm 0.05$ & $1.10^{\mathrm{b}} \pm 0.00$ & $1.16^{\mathrm{ab}} \pm 0.01$ & 0.05 \\
Total ash (\%) & $1.08^{\mathrm{ab}} \pm 0.01$ & $1.05^{\mathrm{c}} \pm 0.00$ & $1.09^{\mathrm{a}} \pm 0.01$ & $1.06^{\mathrm{bc}} \pm 0.00$ & $1.06^{\mathrm{bc}} \pm 0.00$ & 0.005 \\
\hline
\end{tabular}

$a, b, c$ Values in the same row bearing different superscripts are significantly different. SBM, Soybean meal; SHM, Shrimp head meal; P values indicate significance level.

Table 9: Color measurement of different body parts of layer meat (Mean \pm SE) under different dietary treatments

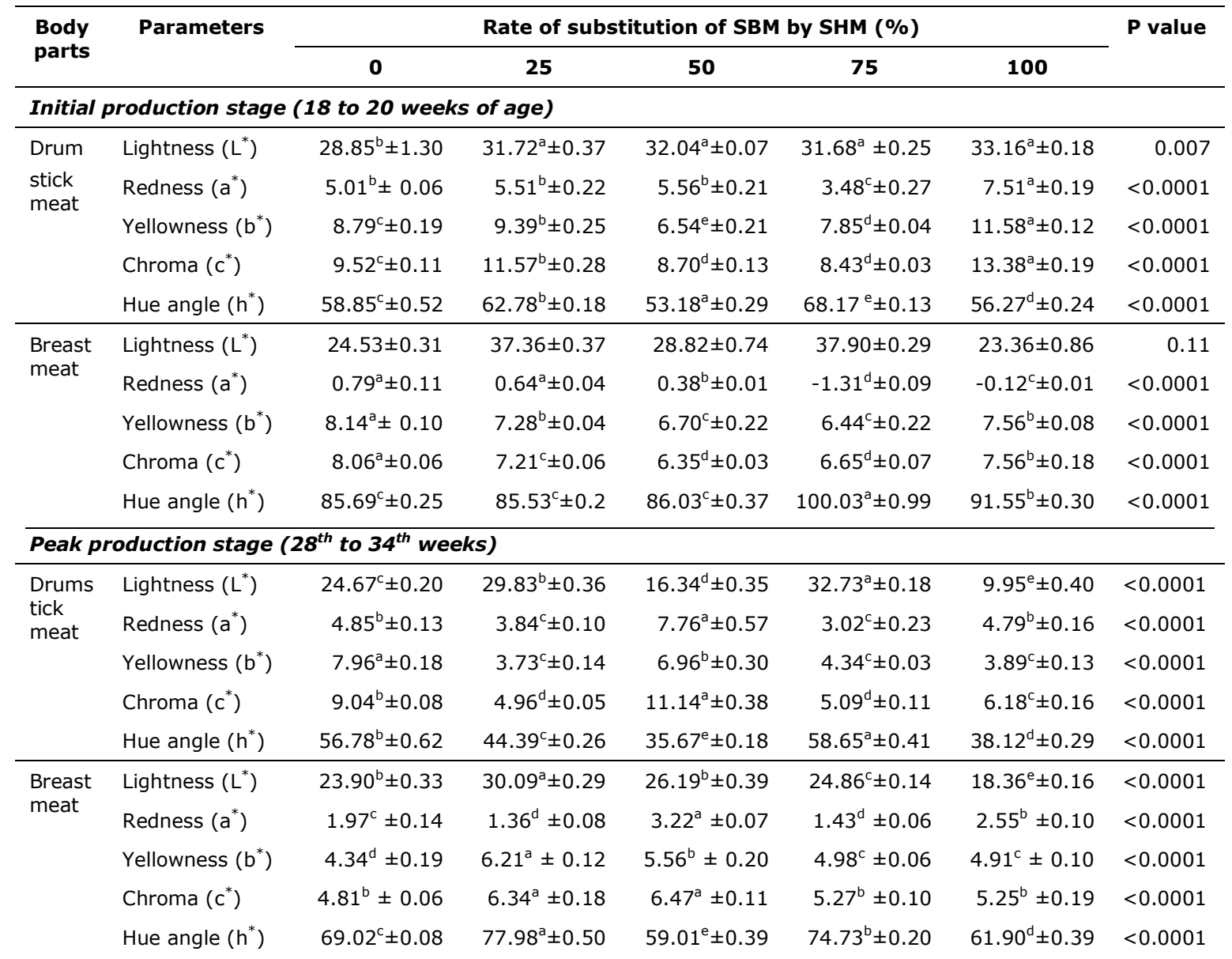

$a, b, c$ Values in the same row bearing different superscripts are significantly different. SBM, Soybean meal; SHM, Shrimp head meal; $P$ values indicate significance level.

Results of a study revealed that shrimp meal had no significant effect on percentage of giblets (liver, gizzard, heart, etc.) yield among the dietary treatment groups ( $p>0.05)$ (Rahman, 2016) but in the present study giblets weight varied significantly due to the inclusion of SHM in the diets. Live weight and dressed weight of laying hens reduced with the increasing levels of SHM indicates that the complete substitution of
SBM contents of the ration by SHM meal is not suitable because it is well established that the high fiber and chitin contents of SHM reduced the digestibility of total ration (Khempaka et al., 2006).

\section{Proximate composition of layer meats}

Crude protein (CP), ether extracts (EE) and total ash contents of chicken drumstick meats were varied among different treatments (Table 8 ). 
Highest CP (\%) was found to be $19.37 \pm 0.36$ in laying hens fed diets substituted SBM at the rate of $75 \%$ by SHM and lowest in complete substituted group. Ether extracts (\%) was found highest in control group $(1.26 \pm 0.03)$ and lowest in $25 \%$ substituted group $(1.08 \pm 0.01)$.

\section{Color measurement of layer meats}

Color measurement indicators like lightness $\left(L^{*}\right)$, redness $\left(a^{*}\right)$, yellowness $\left(b^{*}\right)$, Chroma $\left(c^{*}\right)$ and hue angle $\left(h^{*}\right)$ for drumstick and breast meats of laying hens both at initial and peak production stages are shown in Table 9. Color study revealed that different color indicators of drumstick and breast meats of laying hens at both initial and peak production stages differed significantly among diet groups included different levels of SHM except lightness $\left(L^{*}\right)$ of breast meat at initial production stage (Table 9 ). The values of redness $\left(a^{*}\right)$ for drumstick meat were found highest in complete substituted diet group $(7.51 \pm 0.19)$ at initial production stage and in $50 \%$ substituted group $(7.76 \pm 0.57)$ at peak production stage. Lightness $\left(\mathrm{L}^{*}\right)$ value of breast meat at peak production stage was found highest in hens group fed $25 \%$ substituted diet $(30.09 \pm 0.29)$ and lowest in complete substituted group $(18.36 \pm 0.16)$ at peak production stage.

\section{Conclusion}

From the present findings, it can be concluded that the shrimp head meal (SHM) is a good alternative source of protein in layer ration for good quality eggs and meats. Due to high fiber and chitin contents of SHM, complete substitution of soybean meal (SBM) contents of laying hens ration SHM is not suitable. Comparing with each treatment it was found that substitution at the rate of $25 \%$ of SBM of ration by SHM is suitable for better quality eggs and meats of chicken.

\section{Acknowledgement}

The authors are grateful to the Ministry of Education, People's Republic of Bangladesh for financial support under the "Grants for Advanced Research in Science".

\section{Conflict of interest}

There is no conflict of interest among the authors.

\section{References}

Adeyemi OA (2005). Nutritional evaluation of broiler diets formulated with enriched unpeeled cassava root meal fermented with rumen filtrates. $P h D$ thesis, Department of Animal Production and
Health, University of Agriculture, Abeokuta, Nigeria, pp. 185

Aktar M, M Rashid, MG Azam, MAR Howlider and MA Hoque (2011). Shrimp waste and marine waste as substitutes of fish meal in broiler diet. Bangladesh Journal of Animal Science 40(12):18-22.

URL:https://www.researchgate.net/publication/ 265948582

AMSA (1995). American Meat Science Association. Research guidelines for cookery, sensory evaluation and tenderness measurements of fresh meat. Chicago: National Livestock and Meat Board, IL.

Anderson DM, JL Maclssac, MA Daniel, TL Mackinnon and KL Budgell (2008). Evaluating the effects of crab meal, Carophyll Red $\AA$, and Carophyll Yellow ${ }^{\circledR}$ in laying hen diets on egg yolk pigmentation and production performance. Canadian Journal of Animal Science 88:637640.

URL:

https://www.nrcresearchpress.com/doi/pdf/10.4 141/CJAS08063

Ani AO and AU Okorie (2009). Response of broiler finishers to diets containing graded levels of processed castor oil bean (Ricinus communis $\mathrm{L}$ ) meal. Journal of Animal Physiology and Animal Nutrition 93(2):157-164. DOI: https://doi.org/10.1111/j.14390396.2007.00796.x

AOAC (2005). Official Method of Analysis. 18th Edition, Association of Official Analytical Chemists, Washington, DC.

Fanimo AO, E Mudama, TO Umukoro and $\mathrm{OO}$ Oduguwa (1996). Substitution of shrimp waste meal for fish meal in broiler chicken ratio. Tropical Agriculture 73(3):201-205.

Gernat AG (2001). The effect of using different levels of shrimp meal in laying hen diets. Poultry Science 80(5):633-636. URL:https://www.sciencedirect.com/science/arti cle/pii/S003257911941136X

Hossain, MI, FH Shikha and AD Sharma (2018). Waste management status of shrimp processing plants of south and south-west region of Bangladesh. Journal of Environmental Science and Natural Resources 11(1\&2):73-81. URL:https://www.banglajol.info/index.php/JESN R/article/view/43374

Hunterlab (1996). Applications note: CIE L* a* b* color scale, Vol. 7, Virginia: Hunterlab.

Khempaka S, M Mochizuki, K Koh and Y Karasawa (2006). Effect of chitin in shrimp meal on growth performance and digestibility in growing broilers. The Journal of Poultry Science 43(4):339-343. https://doi.org/10.2141/jpsa.43.339

Kirkpinar $F$ and $Z$ Acikgoz (2018). Feeding. In: Animal Husbandry and Nutrition, Yucel, B. and Turgay Taşkin, T. Edited. Chapter 5. DOI: 10.5772/intechopen.78618.

URL:https://www.intechopen.com/books/animal -husbandry-and-nutrition/feeding 
Yeasmin et al. (2021) Bang. J. Anim. Sci. 50 (1):12-21

Rahman ABMM (2016). Nutritional studies on the utilisation of shrimp by-products as a potential protein source for chicken feed. PhD thesis, Shinshu University, Japan. URL: https://www.google.com/url?sa $=$ t\&rct $=j \& q=\& e s$ $r c=s \&$ source $=$ web\&cd $=\& c a d=r j a \& u a c t=8 \& v e d=$ 2ahUKEwj4jN-

EzZ7qAhXnXDgGHYHcDLwQFjAAegQIAXAB\&url= https\%3A\%2F\%2Fsoar-

ir.repo.nii.ac.jp\%2F\%3Faction\%3Drepository ur i\%26item_id\%3D18544\%26file_id\%3D72\%26fil e_no\%3D4\&usg=AOvVaw1rvsKXLIXUYwUcSXeM $\mathrm{zzYj}$
Rahman M and K Koh (2014). Nutritional quality and in vitro digestibility of shrimp meal made of heads and hulls of black tiger (Penaeus monodon), white leg (Litopenaeus vannamei) and Argentine red (Pleoticus muelleri) shrimps. Journal of Poultry Science 51:411-415. DOI:https://10.2141/jpsa.0140002

SAS (2009). Statistical Analysis System, Computer Software, Version 9.1.3: Statistics SAS Institute Inc. Cary, NC 27513, NC27513, USA.

Singh RA (1990). Poultry Production. Third edition, Kalyani Publishers, New Delhi, India, pp.237257. 